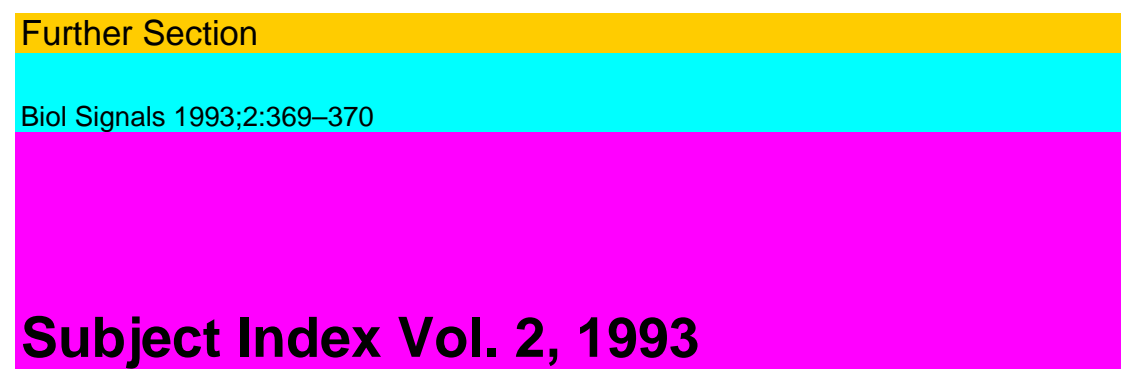

Adrenal zona glomerulosa cell 352 Airway muscle 272

Aldosterone 352 Angiotensin II 106,166, 352 Aplysia 95

Apotransferrin 117 L-Arginine analogues 37 Asthma 305

Bloodvessels 177 Buccal ganglion 95

Calcium antagonist 241

channel(s) 241,284

- metabolism 313

- oscillations 1

- pump inhibitors 293

- signal 272

- signalling 305

- stores 272

Captopril, arterial blood pressure

106 Cardiovascular neurons 106 Carotid sinus nerve 16 Cation 207

Cecum 181 Cell activation 117

- growth 117

Central nervous system 177

Cerebral artery 221

Chorionic gonadotropin 155

Cimetidine 37

Colon 181

Continuous, on-line, real-time

power spectrogram 45 Corticosterone 166 Cyclic adenosine 3',5'-

monophos-

phate 166

- - -, other intracellular

signals 313 Cyclopiazonic acid 263,293 CytosolicCa ${ }^{2+} 253,352$

- -, oscillations, computer

simulation 1

- B sensitivity 253

Deoxycorticosterone 352 Desferal 37 Dexamethasone 352 Diabetes

305 Diferric transferrin 117 2,5-Di-(tert-butyl)-l ,4-benzohy-

droquinone 293 Diurnal rhythm 146,181,207,

228 Dopamine 16 Double discharge units 106 Duodenum 181

Electrical microstimulation, blood

pressure changes 106 Endothelium-derived relaxing

factor 37 Enteric nervous system 136 Esophagus 181 Excitation-

contraction coupling

272 
Feedback modulation 84

Gastropods 95 Glossopharyngeal nerve 16 Guanine nucleotides 27

Guanosine-triphosphate-binding

proteins 241 Gut 177

Heart 177

High-performance liquid chromatography 359

Hirschsprung's disease, animal model 136

Histamine, edema 37

6-Hydroxymelatonin 359

Hypertension 207, 305

\section{Ileum 181}

Information theory 57 In situ hybridization histochemistry 77 Insulin, other growth factors 313 P2-Integrins 126

Intracellular calcium release 241

- $\quad$ free $\mathrm{Ca}^{2+}$ level 84

- - - signalling Vlfli.i 2-[ ${ }^{125}$ I]iodomelatonin 27,221 1 binding sites

177,181,194 Iodide metabolism 313

Ion channels 263 Iron 117

Jejunum 181

Kidney 177

Lung 177

Macrophage 84

Male quail, 2-[ ${ }^{125}$ I]iodomelatonin

binding 146 Melatonin 27,177,194,199,

207

- pharmacokinetics 359

- receptor(s) 146, 181,194,207,228

Membrane transduction 305 Mepyramine 37 Mesenteric artery, dog

263 Methylene blue 37 Mutant rat 136 Myenteric plexus 136

Myogenic response 241

Neural integration 95 Neuromessenger 57 Neuronal communication 57

Neuropeptides 313 Neurotransmitter 57 Nicotine 199 Nitric oxide 37

Norepinephrine 16

- release 199

Opsonized zymosan 126 Oscillatory calcium waves 1

Paracrine secretion 181 Parasympathetic nerves 313

Perifusion, human placental cell

population 155 Permeabilized muscle 253 Photoperiod 146,228 Pineal gland

$27,181,207$

- melatonin 146,313Pituitary hormones 313Placental hormones 313Postsynaptic

potentials 95Potassium 352Presynaptic receptors 199Prostaglandin G/H synthase gene expression 77 Protein kinase C 84,241

Quantitative autoradiography 221 


\section{Rat brain 77}

- $\quad$ vas deferens 199 Receptor downregulation 146

- $\quad$ subtypes 27 Renal system 207 Renin 207

Renin-angiotensin system 166 Retina 177

Rostral ventrolateral medulla 106 Ryanodine 263, 284

Sarcoplasmic reticulum 284, 293 Scanning electron microscopy 136 Seasonal changes

228 Serotonin, edema 37 Signal transduction 1, 84,117 Single discharge units 106

- neuron 45

Smooth muscle(s) 253,293,305 Spontaneously hypertensive rat

106 Stochastic model, cytosolic

calcium oscillators 1 Stress 166

Stretch-induced contraction 241 Submucosal plexus 136 6-Sulfatoxymelatonin 359

Superoxide 126

- dismutase 37Sympathetic nerves 313Synaptic connections 95

- input distribution, cross-correlation study 95

Systemic arterial pressure 45

Tail artery, melatonin receptors 221

Thapsigargin 263,293

Thermoregulation 221

Thymidine uptake 117

Thyrocyte proliferation, thyroid growth 313

Thyroid tumorigenesis, growth-affecting immunoglobulins 313

Thyrotropin 313

Transduction system 57

Transmembrane calcium influx 241

Trophoblast 155

Tyrosine hydroxylase 16

Ureter, guinea pig 263 Urine output 207

Vascular smooth muscles 284

- tissue 241

Vas deferens 177,194

3. 370

Subject Index 